\title{
Adherence to the Mediterranean dietary pattern among Balearic Islands adolescents
}

\author{
Elisa Martínez, Rosa Llull, Maria del Mar Bibiloni, Antoni Pons and Josep A. Tur* \\ Research Group on Community Nutrition and Oxidative Stress, University of the Balearic Islands, Guillem Colom Building \\ Campus, E-07122 Palma de Mallorca, Spain
}

(Received 19 March 2009 - Revised 17 November 2009 - Accepted 24 November 2009 - First published online 21 January 2010)

\begin{abstract}
The aim of the present work was to assess the prevalence of the Mediterranean dietary pattern (MDP) in Balearic Islands adolescents, and sociodemographic and lifestyle factors that might determine adherence to the MDP. A cross-sectional nutritional survey was carried out in the Balearic Islands between 2007 and 2008. A random sample ( $n$ 1231) of the adolescent population (12-17 years old) was interviewed. Dietary questionnaires and a general questionnaire incorporating questions related to socio-economic status, parental education level and lifestyle factors were used. Dietary habits were assessed by means of two $24 \mathrm{~h}$ recalls and a quantitative FFQ. Adherence to the MDP was defined according to a score constructed considering the consumption of nine MDP characteristic components: high MUFA:SFA ratio, moderate ethanol consumption, high legumes, cereals and roots, fruits, vegetables and fish consumption, and low consumption of meat and milk. Then, socio-demographic, lifestyle and health status variables that could determine a higher or ower adherence were assessed. The mean adherence was 57.9 (SD 8.9 ) \% and the median adherence was $57.3 \%$. Half of the Balearic Islands adolescents $(50.5 \%)$ showed an adherence to the MDP comprised between 52.7 and $62.8 \%$. By multivariate analyses, a high maternal level of education, increased physical activity, reduced alcohol intake and abstinence from smoking were independent associations of better adherence to the MDP. The promotion of not only the MDP but also the Mediterranean lifestyle, including greater physical activity, should be reinforced among the Balearic younger generations.
\end{abstract}

Nutrition surveys: Mediterranean dietary pattern: Balearic Islands: Mediterranean region: Adolescents

Adolescence is a time of rapid physiological, psychological and social development influencing nutrient needs and an individual's ability to supply those needs. Sociocultural factors, i.e. parental occupational status, maternal level of education, cultural and/or religious habits, the role of family, patterns of beauty, etc., are factors that have a strong influence on eating habits in this age group and, hence, their nutrient intake $^{(1)}$. Therefore, in light of the high nutritional needs during adolescence, findings that increase knowledge about these factors could influence the development of healthy eating patterns that may continue into adulthood and decrease inadequate dietary intakes reported by many adolescents.

The traditional Mediterranean dietary pattern (MDP) is characterised by a high intake of vegetables, legumes, fruits and nuts, and cereals (which in the past were largely unrefined), a high intake of olive oil but a low intake of saturated lipids, a moderately high intake of fish (depending on the proximity of the sea), a low-to-moderate intake of dairy products (and then mostly in the form of cheese or yoghurt), a low intake of meat and poultry and a regular but moderate intake of ethanol, primarily in the form of wine and generally during meals ${ }^{(2)}$. The traditional MDP has been associated with a reduced risk of several non-transmittable chronic diseases and with prolonged survival; hence, the MDP has been promoted as a model for healthy eating ${ }^{(3-5)}$.

Epidemiological evidence suggests that dietary patterns in the Mediterranean countries are changing rapidly, with an increased consumption of animal products and saturated fat and a decline of intake of basic foodstuffs based on vegetables ${ }^{(6)}$. Reasons for this development can be found in the substantial socioeconomic changes throughout all of Europe over the past 40 years. All the recent nutritional surveys carried out in Spain in different groups of the population confirm a progressive departure from the traditional Mediterranean diet, mainly in younger generations ${ }^{(7-11)}$. The most important food sources of Spanish children aged 6-7 years were white bread (13.4\% of carbohydrate), olive oil (13.8\% of lipids), whole milk $\left(10.2 \%\right.$ of protein) and French fries $(30.4 \% \text { of PUFA })^{(12)}$. All these changes observed in the Mediterranean diet involve deterioration not only of the macronutrient but also the micronutrient content of the Mediterranean diet and therefore its benefits on health ${ }^{(13)}$.

The food pattern of the adult population of the Balearic Islands is in a transitional state characterised by the loss of the traditional MDP towards a more Western dietary pattern, with increased consumption of animal products 
and saturated fat and detriment of basic foodstuffs on a vegetable basis ${ }^{(12,14)}$.

In spite of several epidemiological studies that have evaluated the adherence of a population to the $\mathrm{MDP}^{(15)}$, to our knowledge, few approaches have tested the adherence to the Mediterranean diet among young populations, mainly in Spanish, Greek and Cypriot youngsters. In 1998-2000, only $46.4 \%$ of Spanish children and young people (2-24 years) had an optimal Mediterranean $\operatorname{diet}^{(16)}$. In 2007, only $11.3 \%$ of Greek children and $8.3 \%$ of adolescents reported eating habits following the traditional $\mathrm{MDP}^{(17)}$. In 2004, Spanish adolescents (12-17 years) of Guadalajara (Spain) showed $42.8 \%$ of mean adherence to the $\mathrm{MDP}^{(18)}$. In 2004-2005, $6.7 \%$ of Cypriot pre-adolescents reported high adherence to the $\operatorname{MDP}^{(19)}$.

The aim of the present study was to assess the prevalence of the MDP in the Balearic Islands adolescents, and sociodemographic and lifestyle factors that might determine adherence to the MDP.

\section{Methods}

\section{Study design}

The study is a population-based cross-sectional nutritional survey carried out in the Balearic Islands between 2007 and 2008.

\section{Selection of participants, recruitment and approval}

The target population consisted of all inhabitants living in the Balearic Islands aged 12-17 years. The sample population was derived from residents aged 12-17 years registered in the scholar census of the Balearic Islands. The theoretical sample size was set at 1500 individuals in order to provide a specific relative precision of $5 \%$ (type $\mathrm{I}$ error $=0.05$; type II error $=0 \cdot 10$ ), taking into account an anticipated $70 \%$ participation rate. The sampling technique included stratification according to municipality size, age and sex of inhabitants, and randomisation into subgroups, with Balearic Islands municipalities being the primary sampling units, and individuals within the schools of these municipalities comprising the final sample units. The interviews were performed at the schools. The final sample size was 1231 individuals ( $82 \%$ participation). The reasons to not participate were: (1) the subject declined to be interviewed; (2) the parents did not authorise the interview.

\section{General questionnaire}

A questionnaire incorporating the following questions was used: age group; region of origin (defined as being born in the Balearic Islands, East of Spain as representative of the Spanish Mediterranean coast, other parts of Spain and other countries); father's and mother's educational level (grouped according to years and type of education: low, $<6$ years at school; medium, 6-12 years of education; high, $>12$ years of education); parental socio-economic level (based on the occupation of parents and classified as low, medium and high, according to the methodology described by the Spanish
Society of Epidemiology $\left.{ }^{(20)}\right)$. Anthropometric measurements were also obtained.

Physical activity was evaluating according to the guidelines for data processing and analysis of the international physical activity questionnaire ${ }^{(21)}$ in the short form, and its specific modification for adolescents (International Physical Activity Questionnaire A) ${ }^{(22)}$. The specific types of activity assessed were walking, moderate-intensity activities (i.e. physical activity at school) and vigorous-intensity activities (i.e. sport practice), and an additional question about sitting time was using as an indicator variable of time spent in sedentary activity. According to the AVENA (Alimentación y Valoración del Estado Nutricional en Adolescentes; Food and Assessment of Nutritional Status of Adolescents) study $^{(23)}$, the questionnaire also included information on hours per day of television viewing, computer use, video games, another leisure time physical activity practice and typical sleep duration to the nearest $10 \mathrm{~min}$. The physical activity assessed by the international physical activity questionnaire was correlated with physical activity level (PAL) according to the estimation of the individualised activity coefficient. Each subject was classified taking into account its PAL value ${ }^{(24)}$ as sedentary (PAL $\geq 1.0<1.4$ ); low active (PAL $\geq 1.4<1.6$ ); active (PAL $\geq 1.6<1.9$ ); very active (PAL $\geq 1 \cdot 9$ ). Each subject was then classified into no active (sedentary or low active) and active (active or very active).

Information about smoking habits and alcohol consumption was collected as described: smoking habit (no; yes; occasionally, <1 cigarette/d); alcohol consumption (no; frequently; occasionally, $<1$ drink/week).

\section{Dietary questionnaire}

Dietary questionnaires included two non-consecutive $24 \mathrm{~h}$ diet recalls and a semi-quantitative FFQ previously used in a pilot study on Balearic Islands adolescents ${ }^{(1)}$. The FFQ was previously validated ${ }^{(25)}$ and applied to other studies and surveys over the Spanish population ${ }^{(11,14,26,27)}$. The FFQ, which asked the subject to recall average use over the past year, consisted of 145 items (118 of the original validated FFQ plus the most characteristic Balearic Islands foods in order to make easy the interviewee answer), and arranged by food type and meal pattern. Frequency of food consumption was based on times that food items were consumed (per day, week or month). Consumption $<1 /$ month was considered no consumption. Daily consumption (g) was determined by dividing the reported amount of the intake by the frequency $(d)$. The period of consumption of seasonal items was also considered. Edible fractions of foods were recorded in the database ${ }^{(25-27)}$.

To avoid bias brought on by day-to-day intake variability, the questionnaires were administered homogeneously from Monday to Sunday. Well-trained dietitians administered the recalls and verified and quantified the food records. To estimate volumes and portion sizes, the household measures found in the subjects' own homes were used. Conversion of food into nutrients was made using a self-made computerised program based on Spanish ${ }^{(28,29)}$ and European $^{(30)}$ food composition tables and complemented with food composition data available for Majorcan food 
items $^{(31)}$. Identification of misreporters: an energy intake:BMR ratio $<0.92$ (men) and $<0.85$ (women) was considered to represent underreporting ${ }^{(32)}$, and an energy intake:BMR ratio $\geq 2.4$ as overreporting ${ }^{(33,34)}$.

\section{Mediterranean dietary pattern}

The MDP has been defined according to a previously defined score indicating the degree of adherence to the traditional Mediterranean diet ${ }^{(2,11,35)}$. This Mediterranean dietary score was converted to relative percentage of adherence using a previously described method ${ }^{(9)}$ that will now be briefly summarised.

An energy-adjusted value was obtained for each individual for the daily consumption of legumes, cereals and roots (including bread and potatoes), fruit (including nuts), vegetables, fish, meat (and meat products) and whole milk (and whole milk products). The alcohol consumption in adolescents must be null, and values above the reference indicate a consumption alcohol on the part of adolescents. Information about the consumption of all the food items was obtained from the FFQ.

All the values were standardised as a $Z$ value. A $Z$ score expresses the difference between the individual's measurement and the mean value of the reference population (in this case, the study population) as a proportion of the SD of the reference population (observed intake - mean intake/SD). The total Mediterranean dietary score was computed by adding up all the $Z$ scores obtained for the favourable or 'more Mediterranean' dietary components (legumes, cereals and roots, fruit, vegetables, fish and MUFA:SFA ratio) and substracting the $Z$ value obtained from the consumption of meat, whole milk (mainly high in fat) and alcohol (in adolescents):

$$
\begin{aligned}
\Sigma Z_{i}= & Z_{\text {legumes }}+Z_{\text {cereals and roots }}+Z_{\text {fruit }}+Z_{\text {vegetables }}+Z_{\text {fish }} \\
& +Z_{\text {MUFA:SFA }}-Z_{\text {meat }}-Z_{\text {whole milk }}-Z_{\text {alcohol }} .
\end{aligned}
$$

The Mediterranean dietary score was converted to relative percentage of adherence using the range of values of

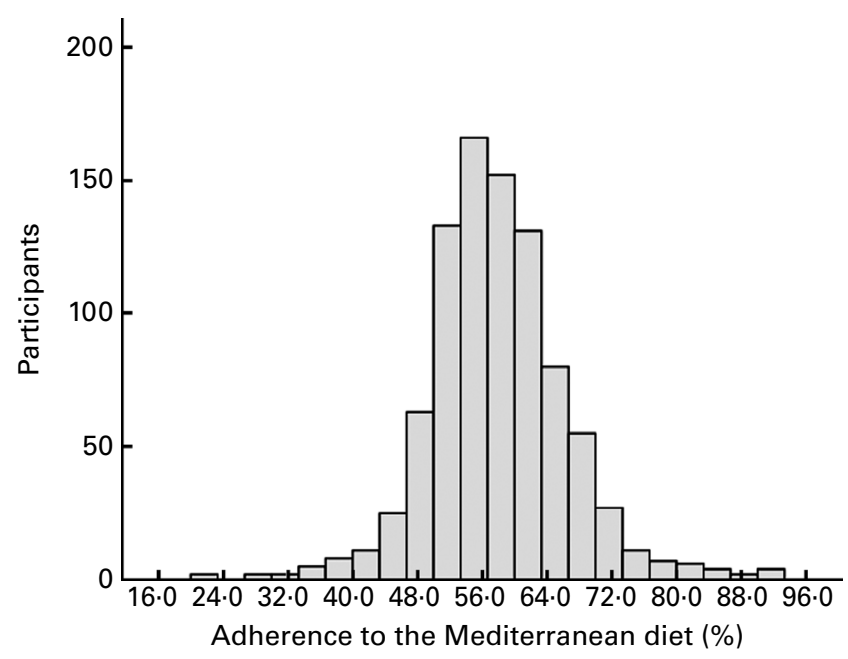

Fig. 1. Distribution of percentage of adherence to the Mediterranean dietary pattern among the Balearic Islands adolescents. the sample. This percentage ranged from 100 (maximum adherence) to 0 (minimum adherence):

$$
\begin{aligned}
\text { Adherence }\left(\text { percentage }_{i}\right)= & \left(\Sigma Z_{i}-\Sigma Z_{\min }\right) \times 100 /\left(\Sigma Z_{\max }\right. \\
& \left.-\Sigma Z_{\min }\right) .
\end{aligned}
$$

Once the percentage of adherence to the MDP was calculated, the variables that could determine a higher or lower adherence were assessed.

\section{Statistics}

Analyses were performed with Statistical Package for the Social Sciences version 16.0. All tests were stratified by sex. Mean adherence and SD were calculated. Quartile values of adherence to the Mediterranean diet were calculated in order to find the group of the population with the lowest adherence percentage (percentage below the lower quartile value) and those with the highest adherence (percentage of adherence above the upper quartile value). To further study the sociodemographic and lifestyle differences between those with the lowest and highest adherence (in order to assess which variables better determined a high or low adherence), logistic regression models were used with the calculations of corresponding age- and sex-adjusted OR and $95 \% \mathrm{CI}$. The level of significance was established for $P$ values $<0.05$.

\section{Ethics}

The present study was conducted according to the guidelines laid down in the Declaration of Helsinki, and all procedures involving human subjects were approved by the Balearic Islands Ethics Committee. Written informed consent was obtained from all subjects and their parents or legal tutors.

\section{Results}

A total of 1231 individuals formed part of the present study. Underreporters ( $n$ 246) and overreporters ( $n$ 24) were excluded from the analysis of dietary patterns in order to avoid respondent bias usually present in recall dietary methods.

Fig. 1 shows that the distribution of percentage of adherence to the MDP roughly follows a normal distribution with a little SD. The mean adherence was 57.9 (SD 8.9)\% and the median adherence was $57.3 \%$. The lowest adherence to the MDP was defined as percentage of adherence below the lower quartile (52.7\%), and the highest adherence was defined as percentage of adherence above the upper quartile $(62 \cdot 8 \%)$.

Means and standard deviations of the percentage of adherence to the MDP according to socio-demographic and lifestyle factors are shown in Table 1, together with age- and sex-adjusted OR for a low adherence, to find the factors with high risk of low adherence to the MDP.

Girls' percentage of adherence to the MDP was greater than boys' ( 58.7 and $56.9 \%$, respectively), and this difference was statistically significant after adjusting for age. An inverse association can be observed between age and the risk of low adherence to the MDP, although this difference is not statistically significant. The trend of decreasing adherence to the MDP with age happened in boys, whereas in girls the 
Table 1. Percentage of adherence and risk of a low adherence according to socio-demographic and lifestyle variables (Mean values and standard deviations; OR and $95 \% \mathrm{Cl}$ )

\begin{tabular}{|c|c|c|c|c|c|}
\hline \multirow[b]{2}{*}{ Socio-demographic and lifestyle variables } & \multicolumn{2}{|c|}{$\begin{array}{l}\text { Percentage of } \\
\text { adherence }\end{array}$} & \multicolumn{3}{|c|}{ Risk of low adherence ${ }^{*}$} \\
\hline & Mean & SD & Age- and sex-adjusted OR & $95 \% \mathrm{Cl}$ & $P$ value \\
\hline \multicolumn{6}{|l|}{ Sex } \\
\hline Male & $56 \cdot 9$ & 8.9 & 1.67 & $1 \cdot 18,2 \cdot 36$ & 0.004 \\
\hline Female & $58 \cdot 7$ & $8 \cdot 8$ & 1.00 (ref.) & & \\
\hline \multicolumn{6}{|l|}{ Age group (years) } \\
\hline $12-13$ & 58.5 & $9 \cdot 3$ & 0.83 & $0.50,1.38$ & 0.477 \\
\hline $14-15$ & $58 \cdot 1$ & $8 \cdot 3$ & 0.88 & $0.59,1.31$ & 0.527 \\
\hline $16-17$ & $57 \cdot 0$ & $9 \cdot 6$ & 1.00 (ref.) & & \\
\hline \multicolumn{6}{|l|}{ Place of birth } \\
\hline Mediterranean Spanish regions & 59.9 & $11 \cdot 6$ & 0.87 & $0.32,2.36$ & 0.787 \\
\hline Other Spanish regions & 57.9 & $7 \cdot 1$ & 0.65 & $0.29,1.49$ & 0.313 \\
\hline Other Mediterranean countries & 58.9 & $9 \cdot 4$ & 0.42 & $0.09,2.01$ & 0.280 \\
\hline Other countries & 57.9 & 8.0 & 0.81 & $0.47,1.39$ & 0.439 \\
\hline Balearic Islands & $57 \cdot 8$ & $9 \cdot 0$ & 1.00 (ref.) & & \\
\hline \multicolumn{6}{|l|}{ Educational level† } \\
\hline \multicolumn{6}{|l|}{ Father } \\
\hline Low & $57 \cdot 0$ & $8 \cdot 7$ & 0.77 & $0.43,1.37$ & 0.378 \\
\hline Medium & 57.9 & 8.4 & 0.94 & $0.59,1.50$ & 0.802 \\
\hline High & $59 \cdot 1$ & $9 \cdot 7$ & 1.00 (ref.) & & \\
\hline \multicolumn{6}{|l|}{ Mother } \\
\hline Low & $56 \cdot 3$ & $8 \cdot 1$ & 1.86 & $1.02,3.39$ & 0.042 \\
\hline Medium & $58 \cdot 2$ & $8 \cdot 7$ & 1.09 & $0.67,1.75$ & 0.736 \\
\hline High & $59 \cdot 1$ & 9.4 & 1.00 (ref.) & & \\
\hline \multicolumn{6}{|l|}{ Parental socio-economic status $\ddagger$} \\
\hline Low & $57 \cdot 2$ & $8 \cdot 9$ & $1 \cdot 19$ & $0 \cdot 66,2 \cdot 14$ & 0.570 \\
\hline Medium & $58 \cdot 2$ & $9 \cdot 1$ & 0.93 & $0.57,1.51$ & 0.765 \\
\hline High & $58 \cdot 4$ & 8.6 & 1.00 (ref.) & & \\
\hline \multicolumn{6}{|l|}{ PAL $\$$} \\
\hline Sedentary & $57 \cdot 2$ & $9 \cdot 6$ & $2 \cdot 66$ & $0.92,7.68$ & 0.072 \\
\hline Low active & $57 \cdot 6$ & 7.9 & $2 \cdot 27$ & $1.07,4.81$ & 0.033 \\
\hline Active & $58 \cdot 1$ & 9.9 & 1.88 & $0.91,3.92$ & 0.090 \\
\hline Very active & $58 \cdot 5$ & $7 \cdot 1$ & 1.00 (ref.) & & \\
\hline \multicolumn{6}{|l|}{ Smoking habit|| } \\
\hline Yes & $53 \cdot 3$ & $11 \cdot 3$ & $2 \cdot 24$ & $1 \cdot 09,4.59$ & 0.028 \\
\hline No & 58.4 & 8.4 & 1.07 & $0.74,1.54$ & 0.733 \\
\hline Occasionally & $58 \cdot 0$ & $8 \cdot 8$ & 1.00 (ref.) & & \\
\hline \multicolumn{6}{|l|}{ Alcohol consumption? } \\
\hline No & 58.6 & $8 \cdot 2$ & 0.53 & $0.35,0.80$ & 0.002 \\
\hline Yes & $55 \cdot 3$ & $10 \cdot 7$ & 1.00 (ref.) & & \\
\hline
\end{tabular}

ref., Reference; PAL, physical activity level.

* Multiple logistic regression analysis adjusting for age and sex. Low adherence was defined as a percentage of adherence below the lower quartile $(52.7 \%)$; high adherence was defined as a percentage of adherence above the upper quartile $(62.8 \%)$

$\dagger$ Educational status is grouped according to years and type of education: low ( $<6$ years of education); medium (6-12 years of education); high ( $>12$ years of education).

$\ddagger$ Socio-economic level is based on the occupation of parents.

$\S \mathrm{PAL}$ was determined according to the level of exercise reported by the interviewee during their free time and classified as sedentary $(\mathrm{PAL}<1.4)$; low active $(1.4 \leq \mathrm{PAL}<1.6)$; active $(1.6 \leq \mathrm{PAL}<1.9)$; very active $(\mathrm{PAL} \geq 1.9)$.

$\|$ Smoking habit was classified as no (never); yes ( $>1$ cigarette/d); occasionally $(<1$ cigarette/d)

If Alcohol consumption was classified as no (no glass of alcohol); yes (one or more glass of alcohol per day).

percentages of adherence were similar between groups of age (data not shown). Just $7 \cdot 1 \%$ of the Balearic Islands adolescents showed optimal MDP.

Adolescents with high educational level of parents showed more adherence to the MDP than those with medium and low educational levels. The risk of low adherence in adolescents with low educational level mothers was approximately two times higher than the others. The paternal educational level did not influence the adolescent adherence to the MDP. The parental socio-economic status was not significantly linked to greater or lower adherence to the MDP. Frequent smokers showed the lowest adherence to the MDP and double risk of low adherence to the MDP than occasional and non-smoker adolescents. Non-alcohol drinker adolescents showed higher adherence to the MDP and lower risk of low adherence to the MDP than drinkers. Sedentary and low-active adolescents showed lower adherence to the MDP and lower risk of low adherence to the MDP than active and very active adolescents. There were no differences among adolescents' place of birth.

The distribution of the consumption $(\mathrm{g} / \mathrm{d}$ or $\mathrm{ml} / \mathrm{d}$ ) of the Mediterranean diet components according to low and high adherence to the Mediterranean diet is shown in Table 2. Youngsters with high adherence to the MDP showed higher consumption of more Mediterranean dietary components (cereals and roots, vegetables, fruit, legumes, fish and 
Table 2. Distribution of the consumption $(\mathrm{g} / \mathrm{d}$ or $\mathrm{ml} / \mathrm{d}$ ) of the Mediterranean diet (MD) components according to low and high adherence to the MD

(Mean values and standard deviations)

\begin{tabular}{|c|c|c|c|c|c|}
\hline & \multicolumn{2}{|c|}{$\begin{array}{l}\text { Low adherence } \\
\text { to the MD* } \\
\text { ( } n \text { 543) }\end{array}$} & \multicolumn{2}{|c|}{$\begin{array}{l}\text { High adherence } \\
\text { to the MD } \\
\quad(n 67)\end{array}$} & \multirow[b]{2}{*}{$P$ value } \\
\hline & Mean & SD & Mean & SD & \\
\hline Cereals and roots & $463 \cdot 7$ & $308 \cdot 4$ & $1005 \cdot 8$ & $919 \cdot 8$ & 0.001 \\
\hline Vegetables & $86 \cdot 6$ & $81 \cdot 3$ & 328.9 & 294.5 & 0.001 \\
\hline Fruit & 132.5 & $115 \cdot 0$ & 361.8 & 289.4 & 0.001 \\
\hline Legumes & $16 \cdot 3$ & $17 \cdot 6$ & $66 \cdot 2$ & $101 \cdot 1$ & 0.001 \\
\hline Fish & $17 \cdot 6$ & $15 \cdot 3$ & $73 \cdot 3$ & 90.9 & 0.001 \\
\hline Meat & $235 \cdot 6$ & $197 \cdot 3$ & $230 \cdot 1$ & 209.8 & 0.001 \\
\hline Whole milk (and products) & $543 \cdot 6$ & $399 \cdot 6$ & $487 \cdot 0$ & $265 \cdot 6$ & 0.003 \\
\hline MUFA:SFA & $1 \cdot 2$ & 0.3 & 1.8 & 0.6 & 0.001 \\
\hline Alcohol & 5.5 & $2 \cdot 4$ & 0.8 & $2 \cdot 9$ & 0.016 \\
\hline
\end{tabular}

* Lower quartile ( $<52.5 \%$ adherence to the MD).

†Upper quartile ( $\geq 62.8 \%$ adherence to the MD).

MUFA:SFA ratio) and smaller consumption of meat, whole milk (and products) and alcohol than adolescents with low adherence to the MDP.

\section{Discussion}

In nutritional epidemiology, analyses of individual nutrients and food can ignore important interactions between components of a diet. Moreover, people do not eat isolated nutrients, and then it is always interesting to study the dietary patterns of a population. It is also well known that the Mediterranean diet is an interesting alternative in health promotion because this pattern, and not only its individual nutrients, has been postulated as being protective against several diseases ${ }^{(2,4,9,10)}$. However, despite all the increasing evidence about the benefits of the Mediterranean diet, nowadays we are witnessing the detriment of this diet in many Mediterranean regions towards a more Western-type $\operatorname{diet}^{(36)}$. Therefore, it is interesting to assess the adherence of Mediterranean population to the MDP.

Moreover, studies examining adherence to the MDP among young Mediterranean people are scarce ${ }^{(16-19)}$. So, this is the first time that the adherence to the MDP is assessed in a representative sample of adolescents of the Balearic Islands, a typically Mediterranean region, and contributes to the knowledge on the adherence of Mediterranean adolescents to the MDP.

The adherence to the MDP among adolescents was measured according to a Mediterranean dietary score, computed taking into account the most characteristic component of this dietary pattern ${ }^{(9)}$. The average adherence of adolescents to the MDP was 57.9 (SD 8.9) \%. The observed $\mathrm{SD}$ indicates that the percentage of adherence to the Mediterranean diet is fairly uniform, with low variability across all the study participants. The mean adherence to the MDP showed by Balearic Islands adolescents is higher than that observed in other Spanish adolescents ${ }^{(18)}$, but the percentage of Balearic Islands adolescents that showed optimal MDP is quite similar to other Mediterranean populations $^{(17,19)}$.
It is well known that a high adherence to the MDP means a better diet quality ${ }^{(3-5)}$, and this have been also demonstrated in the present study, since highest consumptions of more Mediterranean dietary components and smallest consumptions of meat, whole milk (and products) and alcohol have been observed in Balearic Islands adolescents with highest adherence to the MDP.

Girls showed higher adherence and lower risk of having low adherence to the MDP than boys. These results agree with a previous study that pointed out that girls were more likely to pay attention to food as a way to influence health and to meet nutritional recommendations than boys ${ }^{(37)}$. It cannot be discarded, however, that girls underreported their sweets and snacks consumption.

The family educational level has a marked effect on family lifestyles and dietary habits ${ }^{(38)}$. In agreement with these previous studies, mother's educational level was also a stronger factor on the dietary habits of youngsters than father's educational level. Accordingly, there was a direct association between adherence to the MDP and the mother's educational level, whereas no effect of father's educational level on the adolescent's adherence to the MDP was observed. Nowadays, the mother is still responsible for the home food in the Balearic Islands, and the mother's educational level is one of the best predictors of the quality of an adolescent's diet. The present results are also in agreement with previous studies carried out in Spanish adults ${ }^{(6-8,10,11,39)}$ that suggested a direct association between low educational level and low consumption of fruits and vegetables, which are characteristic foods of the MDP.

The adoption of alcohol, tobacco and other drug use and misuse typically begins during adolescence and may mean serious health problems in adulthood. In the present study, non-alcohol drinker adolescents showed higher adherence to the MDP and lower risk of low adherence to the MDP than drinkers. Alcohol consumption is a method with which adolescents can enhance social relationships in which they spend much time ${ }^{(40)}$. The Balearic Islands adolescents consumed alcohol, mainly distilled spirits, on weekends (data not shown), which is closer to Scandinavian patterns ${ }^{(41)}$ than to Mediterranean patterns ${ }^{(42)}$. These studies support our 
conclusion that alcohol consumption is a negative factor on the adherence of adolescents to the MDP. Moreover, the traditional alcohol consumption in the MDP is primarily in the form of wine and generally during meals ${ }^{(2)}$, which is quite far from the adolescent pattern of alcohol consumption.

Frequent smokers showed the lowest adherence to the MDP and double the risk of low adherence to the MDP than occasional and non-smoker adolescents. It has been documented that smoking is linked to less healthy dietary habits ${ }^{(43,44)}$. Moreover, a previous study carried out in adult Spanish populations pointed out that smoking was inversely associated with higher adherence to the traditional $\mathrm{MDP}^{(45)}$. These previous works reinforce our conclusion that smoking habit is a negative factor on the adherence of adolescents to the MDP.

Sedentary and low-active adolescents showed lower adherence to the MDP and lower risk of low adherence to the MDP than active and very active adolescents. This association has also been observed in previous studies $^{(6,10,46-49)}$. Healthy lifestyle variables tend to cluster and therefore we could speak about the Mediterranean lifestyle, including a higher level of physical activity, as one aspect associated with the MDP that can be protective against different diseases.

\section{Limitations of the study}

The methodology of the present study has some limitations, as, for example, the $24 \mathrm{~h}$ recalls provide information on food intake, and because the data collection occurs after consumption, this method does not affect an individual's food choices on a given day ${ }^{(50)}$. At least two non-consecutive administrations are necessary to assess usual intakes, to reduce dependency on intake from the previous day and by household food availability ${ }^{(50)}$. Accordingly, we applied two $24 \mathrm{~h}$ nonconsecutive recalls in the present study.

Although $24 \mathrm{~h}$ recalls collect data soon after intake, recalls also have limitations related to memory and bias ${ }^{(50)}$. Many adolescents have difficulties in estimating their consumption of some foods, and the consequence of difficulties in assessing high-energy-dense foods may be that consumed amounts are underestimated, which in turn may have a great effect on the validity of the reported energy intake ${ }^{(51)}$. Underreporting and overreporting are significant contributors to the systematic bias of self-reported dietary assessments, increasing or decreasing estimates of the incidence of inadequate intake, and distorting the relationships between nutrient intake and health. Therefore, there are real shortcomings in dietary studies.

To solve these shortcomings, it has been pointed out ${ }^{(52)}$ that dietary studies should include an internal validation procedure. Biomarkers of energy intake have been suggested to play a useful role in dietary assessment, especially for components of foods that are highly variable within different samples of the same food. However, biomarkers, even when available, also have many limitations ${ }^{(53)}$. Advantages and limitations of each must be carefully considered in any specific application, but the mainstay of nutritional epidemiology will remain assessments of dietary intake.

Physical activity is a complex multidimensional exposure, which is difficult to measure by self-reported questionnaires in epidemiological studies ${ }^{(54)}$. Self-report of physical activity can lead to overreporting of the physical activity due to a social desirability bias, and therefore the number of inactive individuals may be greater than that reported ${ }^{(55,56)}$, especially among children and adolescents, and also among the obese ${ }^{(55)}$. Questionnaires have inherent limitations, mainly because they are subjective in nature. Limitations in the validity of physical activity questionnaires are considered the main reasons for inaccuracies in epidemiological studies.

An extensive range of instruments for measuring physical activity has been reported in the literature, but critical elements in the utility of an instrument to measure physical activity are that it will be relatively inexpensive, cause minimal inconvenience to the participant and be able to be administered with relative ease ${ }^{(57)}$.

\section{Conclusions}

Current adolescents in the the Balearic Islands show $57.9 \%$ of mean adherence to the MDP, but $7 \cdot 1 \%$ of them show optimal MDP. High maternal level of education and physical activity, as well as poor alcohol consumption and smoking habit are promoting factors of adherence of adolescents to the MDP, and hence to the quality of their diet. The promotion of not only the MDP but also the Mediterranean lifestyle, including greater physical activity, should be reinforced among the Balearic younger generations.

\section{Acknowledgements}

The present work received funding from Spanish Ministry of Health and Consumption Affairs (Programme of Promotion of Biomedical Research and Health Sciences, Project 05/ 1276 and 08/1259, and Red Predimed-RETIC RD06/0045/ 1004), Spanish Ministry of Education and Science (FPU Programme, PhD fellowship to Maria del Mar Bibiloni). The authors' contributions were as follows: A. P. and J. A. T. conceived, designed and devised the study. M. d. M. B., E. M., R. L. and J. A. T. collected and supervised the samples. E. M. and J. A. T. analysed the data and wrote the manuscript. A. P. and J. A. T. supervised the study. A. P. and J. A. T. obtained funding. The authors state that there are no conflicts of interest.

\section{References}

1. Tur JA, Puig MS, Pons A, et al. (2004) Associations between sociodemographic and lifestyle factors and dietary quality among adolescents in Palma de Mallorca. Nutrition 20, 502-508.

2. Trichopoulou A, Costacou T, Christina B, et al. (2003) Adherence to a Mediterranean diet and survival in a Greek population. New Engl J Med 348, 2599-2608.

3. Mendez MA, Popkin BM, Jakszyn P, et al. (2008) Adherence to a Mediterranean diet is associated with reduced 3-year incidence of obesity. J Nutr 136, 2934-2938.

4. Trichopoulou A \& Vasilopoulou E (2000) Mediterranean diet and longevity. Brit J Nutr 84, Suppl. 2, S205-S209.

5. Martínez-González MA (2004) The emerging role of Mediterranean diets in cardiovascular epidemiology: monounsaturated fats, olive oil, red wine or the whole pattern? Eur J Epidemiol 19, 9-13. 
6. EPIC Group in Spain (2002) Diferencias sociodemográficas en la adhesión al patrón de dieta mediterránea en poblaciones de España (Sociodemographic differences in dietary pattern adherence to the Mediterranean diet in Spanish populations). Gac Sanit 16, 214-221.

7. Aranceta J (2001) Spanish food patterns. Public Health Nutr 4, 1399-1402.

8. Moreno LA, Sarriá A \& Popkin BM (2002) The nutrition transition in Spain: a European Mediterranean country. Eur $J$ Clin Nutr 56, 992-1003.

9. Sánchez-Villegas A, Martínez JA, De Irala J, et al. (2002) Determinants of the adherence to an 'a priori' defined Mediterranean dietary pattern. Eur J Nutr 41, 249-257.

10. Hu FB (2003) The Mediterranean diet and mortality - olive oil and beyond. New Engl J Med 348, 2595-2596.

11. Tur JA, Romaguera D \& Pons A (2004) Adherence to the Mediterranean dietary pattern among the population of the Balearic Islands. Brit J Nutr 92, 341-346.

12. Royo-Bordonada MA, Gorgojo L, de Oya M, et al. (2003) Food sources of nutrients in the diet of Spanish children: the Four Provinces study. Brit J Nutr 89, 105-114.

13. Serra-Majem L (2001) ¿Más beneficios de la Dieta Mediterránea? (More benefits of the Mediterranean diet?). Nutr Obes 4 , 43-46.

14. Tur JA, Romaguera D \& Pons A (2004) Food consumption patterns in a Mediterranean region: does the Mediterranean diet still exist? Ann Nutr Metabol 48, 193-201.

15. Bach-Faig A, Serra-Majem L, Carrasco JL, et al. (2006) The use of indexes evaluating the adherence to the Mediterranean diet in epidemiological studies: a review. Pub Health Nutr 9, 132-146.

16. Serra-Majem L, Ribas L, García A, et al. (2003) Nutrient adequacy and Mediterranean diet in Spanish school children and adolescents. Eur J Clin Nutr 57, Suppl. 1, S35-S39.

17. Kontogianni MD, Vidra N, Farmaki AE, et al. (2008) Adherence rates to the Mediterranean diet are low in a representative sample of Greek children and adolescents. J Nutr 138, 1951-1956.

18. Fernández-Morales I, Aguilar-Vilas MV, Mateos-Vega CJ, et al. (2009) Quality of the diet of a population of young people of Guadalajara. Nutr Hosp 24, 200-206.

19. Lazarou C, Panagiotakos DB, Kouta C, et al. (2009) Dietary and other lifestyle characteristics of Cypriot school children: results from the nationwide CYKIDS study. BMC Public Health 20, $147-156$.

20. Alvarez C, Alonso J, Domingo A, et al. (1995) La medición de la clase social en ciencias de la salud (Social Class Measurements in Health Sciences). Barcelona: SG-Sociedad Española de Epidemiología.

21. IPAQ - International Physical Activity Questionnaire (2008) Guidelines for data processing and analysis of the International Physical Activity Questionnaire (IPAQ). http://www.ipaq.ki.es/ (accessed October 2008).

22. Hagströmer M, Bergman P, De Bourdeaudhuij I, et al. (2008) Concurrent validity of a modified version of the International Physical Activity Questionnaire (IPAQ-A) in European adolescents: the HELENA study. Int J Obes 32, 542-548.

23. Vicente-Rodriguez G, Rey-López JP, Martín-Mantillas M, et al. (2008) Television watching, videogames, and excess of body fat in Spanish adolescents: the AVENA study. Nutrition 24, 654-662.

24. Food and Nutrition Board, Institute of Medicine of the National Academies (2005) Dietary Reference Intakes. Chapter 12, Physical Activity, pp. 880-935. Washington, DC: The National Academies Press.

25. Martin-Moreno JM, Boyle P, Gorgojo L, et al. (1993) Development and validation of a food frequency questionnaire in Spain. Int J Epidemiol 22, 512-519.
26. Serra-Majem L, Morales D, Domingo C, et al. (1994) Comparison of two dietary methods: 24-hour recall and semiquantitative food frequency questionnaire (in Spanish). Med Clin (Barc) 103, 652-656.

27. Bondia-Pons I, Serra-Majem L, Castellote AI, et al. (2007) Identification of foods contributing to the dietary lipid profile of a Mediterranean population. Brit J Nutr 98, 583-592.

28. Moreiras O, Carvajal A, Cabrera L, et al. (2003) Tablas de composición de alimentos (Food Composition Tables), 7th ed. Madrid: Pirámide.

29. Mataix J, Mañas M, Llopis L, et al. (2004) Tablas de composición de alimentos españoles (Spanish Food Composition Tables), 4th ed. Granada: INTA-Universidad de Granada.

30. Feinberg M, Favier JC \& Ireland-Ripert J (1995) Répertoire géneral des aliments (Food Composition Tables). París: Tec \& Doc Lavoisier.

31. Ripoll L (1992) Cocina de las Islas Baleares (The Balearic Islands Cookery). 5th ed. Palma de Mallorca: L. Ripoll.

32. Livingstone MB \& Black AE (2003) Markers of the validity of reported energy intake. J Nutr 133, 895S-920S.

33. Johansson L, Solvoll K, Bjørneboe GA, et al. (1998) Under- and overreporting of energy intake related to weight status and lifestyle in a nationwide sample. Am J Clin Nutr 68, 266-274.

34. Mendez MA, Wynter S, Wilks R, et al. (2003) Under- and overreporting of energy is related to obesity, lifestyle factors and food group intakes in Jamaican adults. Public Health Nutr 7, 9-19.

35. Trichopoulou A, Kouris-Blazos A \& Wahlqvist ML (1995) Diet in overall survival in the elderly. BMJ 311, 1457-1460.

36. Rumm-Kreuter D (2001) Comparison of the eating and cooking habits of northern Europe and the Mediterranean countries in the past, present and future. Int J Vitam Nutr Res 71, 141-148.

37. Tur JA, Romaguera D \& Pons A (2004) Does the diet of the Balearic population, a Mediterranean-type diet, ensure compliance with nutritional objectives for the Spanish population? Public Health Nutr 8, 275-283.

38. Fernández San Juan PM (2006) Dietary habits and nutritional status of school aged children in Spain. Nutr Hosp 21, 374-378.

39. Ortega RM, Mena MC, Faci M, et al. (2001) Vitamin status in different groups of the Spanish population: a meta-analysis of national studies performed between 1990 and 1999. Public Health Nutr 4, 1325-1329.

40. Tur JA, Puig MS, Pons A, et al. (2003) Alcohol consumption among school adolescents in Palma de Mallorca. Alcohol Alcohol 38, 243-248.

41. Laukkanen ER, Shemeikka SL, Viinamäki HT, et al. (2001) Heavy drinking is associated with more severe psychosocial dysfucntion among girls than boys in Finland. $J$ Adol Health 28, 270-277.

42. Paniagua H, García S, Castellano G, et al. (2001) Consumo de tabaco, alcohol y drogas no legales entre adolescentes y relación con los hábitos de vida y el entorno (Tobacco, alcohol and illegal drug consumption among adolescents, relationship with lifestyle and environment). An Pediat 55, 121-128.

43. Lloveras G, Ribas-Barba L, Ramon JM, et al. (2001) Relación del consumo de alimentos y nutrientes con el hábito tabáquico (Food consumption and nutrient intake in relation to smoking). Med Clin (Barc) 116, 129-132.

44. Schröder H, Marrugat J, Elosua R, et al. (2002) Tobacco and alcohol consumption: impact on other cardiovascular and cancer risk factors in a southern European Mediterranean population. Br J Nutr 88, 273-281.

45. Schröder H, Marrugat J, Vila J, et al. (2004) Adherence to the traditional Mediterranean diet is inversely associated with body mass index and obesity in a Spanish population. $J$ Nutr 134, 3355-3361.

46. Costacou T, Bamia C, Ferrari P, et al. (2003) Tracing the Mediterranean diet through principal components and cluster 
analyses in the Greek population. Eur $J$ Clin Nutr 57 , $1378-1385$.

47. Sánchez-Villegas A, Delgado-Rodríguez M, Martínez-González MA, et al. (2003) Gender, age, socio-demographic and lifestyle factors associated with major dietary patterns in the Spanish Project SUN. Clin Nutr 57, 285-292.

48. Someshwar J, Someshwar S \& Perkins KC (2006) The obese adolescent. Pediatr Ann 35, 180-186.

49. Mariscal-Arcas M, Romaguera D, Rivas A, et al. (2007) Diet quality of young people in southern Spain evaluated by a Mediterranean adaption of the diet quality index-international (DQI-I). Brit J Nutr 98, 1267-1273.

50. Strauss RS \& Mir HM (2001) Smoking and weight loss attempts in overweight and normal-weight adolescents. Int J Obes $\mathbf{2 5}$ $1381-1385$.

51. Gromysz-Kałkowska K, Wójcik K, Szubartowska E, et al. (2002) Taste perception of cigarette smokers. Ann Univ Mariae Curie-Sklodowska 57, 143-154.
52. Fernández PM (2006) Dietary habits and nutritional status of school aged children in Spain. Nutr Hosp 21, 374-378.

53. Paniagua H, García S, Castellano G, et al. (2001) Tobacco, alcohol and illegal drug consumption among adolescents, relationship with lifestyle and environment. An Esp Pediatr 55, 121-128.

54. Waling MU \& Larsson CL (2009) Energy intake of Swedish overweight and obese children is underestimated using a diet history interview. J Nutr 139, 522-527.

55. Willett W (2006) Does the future of nutritional epidemiology depend on biomarkers? No. Sixth International Conference on Diet and Activity Methods Abstracts Book, PL03-02, Copenhagen.

56. Almendingen K, Trygg K \& Pedersen JI (1998) An assessment of the use of simple methods to predict individual energy intakes for intervention studies. Eur J Clin Nutr 52, 54-59.

57. Wareham NJ \& Rennie KL (1998) The assessment of physical activity in individuals and populations: why try to be more precise about how physical activity is assessed? Int J Obes 22, S30-S38. 